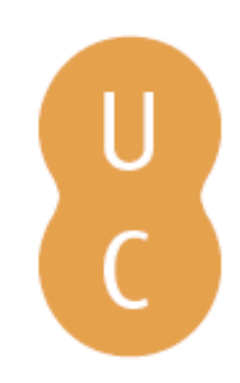

pommalina

\title{
Metodologia em ambiente SIG para localizar dispositivos de auxílio ao ciclista em cidades declivosas
}

Autor(es): $\quad$ Tralhão, Lino; Ribeiro, Nuno; Rodrigues, João Coutinho; Sousa, Nuno

Publicado por: Imprensa da Universidade de Coimbra

URL

persistente: URI:http://hdl.handle.net/10316.2/37099

DOI: $\quad$ DOI:http://dx.doi.org/10.14195/978-989-26-0983-6_46

Accessed : $\quad$ 26-Apr-2023 13:15:05

A navegação consulta e descarregamento dos títulos inseridos nas Bibliotecas Digitais UC Digitalis, UC Pombalina e UC Impactum, pressupõem a aceitação plena e sem reservas dos Termos e Condições de Uso destas Bibliotecas Digitais, disponíveis em https://digitalis.uc.pt/pt-pt/termos.

Conforme exposto nos referidos Termos e Condições de Uso, o descarregamento de títulos de acesso restrito requer uma licença válida de autorização devendo o utilizador aceder ao(s) documento(s) a partir de um endereço de IP da instituição detentora da supramencionada licença.

Ao utilizador é apenas permitido o descarregamento para uso pessoal, pelo que o emprego do(s) título(s) descarregado(s) para outro fim, designadamente comercial, carece de autorização do respetivo autor ou editor da obra.

Na medida em que todas as obras da UC Digitalis se encontram protegidas pelo Código do Direito de Autor e Direitos Conexos e demais legislação aplicável, toda a cópia, parcial ou total, deste documento, nos casos em que é legalmente admitida, deverá conter ou fazer-se acompanhar por este aviso.

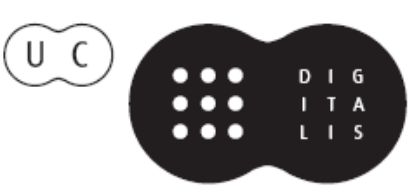




\section{$\forall$ \\ TAS DAS I JORNADAS LUSÓFONAS DE CIÊNCIAS E TECNOLOGIAS DE INFORMAÇÃO GEOGRÁFICA}

Editores

José Gomes dos Santos

Cidália Fonte

Rui Ferreira de Figueiredo

Alberto Cardoso

Gil Gonçalves

José Paulo Almeida

Sara Baptista 


\section{ARTIGO 46 \\ METODOLOGIA EM AMBiente Sig PARA LOCALIZAR \\ Dispositivos DE AUXílio AO CICLISTA EM \\ CIDADES DECLIVOSAS}

TRALHAO, Lino ${ }^{1}$; RIBEIRO, Nuno ${ }^{2}$; COUTINHO-RODRIGUES, João ${ }^{3}$ \& SOUSA, Nuno ${ }^{4}$

${ }^{1}$ Instituto de Engenharia de Sistemas e Computadores (INESC-Coimbra); Rua Antero Quental 199, 3000033 Coimbra, Portugal; Tel: +351 239 851040/9; Fax: +351 239 824692; email: linotralhao@netcabo.pt

2 Departamento de Engenharia Civil da Faculdade de Ciências e Tecnologia da Universidade de Coimbra - Departamento de Engenharia Civil - FCTUC; Rua Luís Reis Santos - Pólo II, 3030-788 Coimbra, Portugal; Tel: +351 239 797135; Fax: +351 239 797123; email: nuno_r_@hotmail.com

3 Departamento de Engenharia Civil da Faculdade de Ciências e Tecnologia da Universidade de Coimbra - Instituto de Engenharia de Sistemas e Computadores (INESC-Coimbra) - Departamento de Engenharia Civil - FCTUC; Rua Luís Reis Santos - Pólo II, 3030-788 Coimbra, Portugal; Tel: +351 239 797145; Fax: +351239 797123; email: coutinho@dec.uc.pt

${ }^{4}$ Departamento de Ciências e Tecnologia da Universidade Aberta Instituto de Engenharia de Sistemas e Computadores (INESC-Coimbra) Delegação de Coimbra da Universidade Aberta; Rua Alexandre Herculano, n. ${ }^{\circ}$ 52, 3000-019 Coimbra, Portugal; Tel: +351 300 001590; Fax: +351 300 001599; email: nsousa@uab.pt

\section{RESUMO}

Preocupações de sustentabilidade relacionadas com o uso intensivo de energia, e o congestionamento de tráfego, têm levado decisores a procurar soluções alternativas, e à emergência de uma mudança para modos de transporte suaves/ativos. A bicicleta é um modo de transporte suave e muito eficiente. No entanto, há também fatores dissuasores do uso da bicicleta - entre estes, é reconhecida a importância do declive na escolha deste modo. Uma das formas de contornar esta limitação consiste na construção de dispositivos mecânicos de auxílio (e.g. elevadores, teleféricos). Apresenta-se neste trabalho uma metodologia que, usando tecnologia SIG, ajuda a identificar potenciais locais para a colocação desses dispositivos, de forma a não só minorar a extensão e número destes, mas também conseguir uma maior permeabilidade da cidade à bicicleta. 
A metodologia, baseada essencialmente na obtenção de áreas de serviço adequadas, permitirá também identificar arcos críticos à conexão ciclável da rede viária urbana Acompanha a abordagem um estudo de caso para a cidade de Coimbra, Portugal, onde, para além da aplicação da metodologia, se considera um circuito para bicicletas e se estuda a permeabilidade ciclável da rede com e sem elevadores, com o circuito, bem como formas de acesso ao local mais importante da cidade.

\title{
PALAVRAS-CHAVE
}

Bicicletas, Sistemas de informação geográfica, Localização, Sustentabilidade, Elevadores para bicicletas.

\section{GIS ENVIRONMENT METHODOLOGY TO LOCATE CYCLING AID DEVICES IN HILLY CITIES}

\begin{abstract}
Sustainability worries related to the intensive use of energy by automobiles, and traffic congestion issues, have encouraged decision makers to look for alternative solutions and to an emerging shift towards soft/active transport modes. The bicycle, a very efficient mode of transport, is one such soft travel mode. Cycling however also has its deterrents among these, it is recognized that slopes play an important role in influencing the choice for this mode. One of the ways to mitigate this limitation consists in constructing hard aid devices (e.g. elevators). In this work a methodology is presented that uses GIS technology to identify potential locations for these connecting devices, such that not only their span and number are minimized but also a greater cycling permeability for the city is acheived. The methodology, which is based in obtaining suitable service areas, will also pinpoint arcs that are critical to the cyclable connectivity of the urban network. A case study for the city of Coimbra, Portugal, is presented, where along with the methodology's application, a bicycling circuit is considered and the network's cycling permeability is studied, with and without devices, with the circuit, as well as means of accessing the city's most important place.
\end{abstract}

\section{KEYWORDS}

Bicycles, Geographical information systems, Location, Sustainability, Bicycle elevators.

\section{INTRODUÇÃO}

A emergência do transporte automóvel no pós-guerra, alimentada pela abundância de petróleo barato e políticas públicas favoráveis, 
levou, em última análise, à sobre- expansão urbana, ineficiências ligadas à congestão de tráfego e consumo de energia e preocupações ambientais. Estas questões têm-se tornado centrais para os decisores políticos, visto ser cada vez mais evidente que o status quo pode não ser sustentável a longo prazo. A bicicleta é um modo de transporte que pode ser adotado na maioria das cidades. Combina a disponibilidade do automóvel com alta eficiência, baixa taxa de congestão (uma bicicleta é aproximadamente igual a 0.23 automóveis, WANG et al. (2008)), benefícios de saúde (WOODCOCK et al., 2007) e brevidade de viagem para baixas distâncias (é competitiva com o automóvel para distâncias até $5 \mathrm{~km}$ (COMISSÃO EUROPEIA, 2000)).

As vantagens da bicicleta não passaram despercebidas aos decisores políticos, que têm levado a cabo ações de fomento do uso da bicicleta nas últimas décadas, um pouco por todo o mundo, mas especialmente na Europa (ver p.ex. BYPAD, 2008). Em paralelo com estas iniciativas, foi feita pesquisa no sentido de saber que fatores influenciam a escolha de uma pessoa pela bicicleta e o que determina os trajetos que ela toma (RIETVELD \& DANIEL, 2004; WARDMAN et al., 2007; PARKIN et al., 2007; PARKIN et al., 2008; PARKIN \& KOOREY, 2012; BROACH et al., 2012). Foram também desenvolvidos modelos para ciclovias, por forma a otimizar a sua atratividade para os ciclistas (SUZUKI et al., 2012). O sucesso destes esforços combinados oscilou entre moderado a considerável no que toca ao aumento da percentagem da bicicleta como modo de transporte (PUCHER et al., 1999; PUCHER \& BUHELER, 2008; PUCHER et al., 2011; CERVERO et al., 2012) e chamou a atenção de decisores em outras cidades e países. No entanto, reproduzir o sucesso de tais iniciativas requer um planeamento cuidadoso, não apenas por questões económicas, mas principalmente porque existem uma série de situações dissuasoras do uso da bicicleta.

Um desses dissuasores é o relevo, que é sabido ter um forte impacto negativo na propensão para o uso da bicicleta nas deslocações diárias (RIETVELD \& DANIEL, 2004; PARKIN et al., 2008). Estudos de aptidão da cidade à bicicleta devem portanto incluir uma componente de relevo, principalmente se a cidade for declivosa. Apresenta-se neste artigo 
uma metodologia que permite ao decisor avaliar a aptidão topográfica de uma cidade para a circulação em bicicleta (aptidão ciclável, ou ciclabilidade) e identificar possíveis melhorias a essa aptidão. Apesar de existirem cidades com dispositivos de auxílio em funcionamento (p.ex. o sistema TRAMPE em Trondheim, Noruega e elevadores em San Sebastian, Espanha), tanto quanto é do nosso conhecimento não existem na literatura métodos com o carácter sistemático do aqui apresentado, razão pela qual cremos que a presente contribuição se reveste de particular importância. Os resultados que gera podem subsequentemente ser usados em modelos existentes de planeamento de trajetos ou circuitos de bicicleta.

A metodologia aqui apresentada consiste de quatro procedimentos: classificação da rede viária, análise de ciclabilidade, análise de permeabilidade, desembocando finalmente na identificação de possíveis localizações de dispositivos de auxílio. Na classificação, um Sistema de Informação Geográfica (SIG) da cidade é usado para discriminar os arcos da rede viária baseando-se no declive destes. Este procedimento, que apresentamos na secção 2.1 juntamente com a análise de ciclabilidade, é basilar para o resto da metodologia e é feito no contexto de um caso de estudo, a cidade declivosa de Coimbra, Portugal. Na análise de permeabilidade, conceito definido e discutido na secção 2.2, áreas de serviço para o uso da bicicleta são calculadas no SIG. Isto permite identificar desconexões na rede ciclável derivadas do relevo, e analisar a sua extensão. Na secção 3 a localização de dispositivos de auxílio é discutida, os quais contribuiriam para suavizar (ou mesmo eliminar) desconexões da rede ciclável e assim melhorar a ciclabilidade global da cidade. Na secção 4 os resultados para Coimbra são agrupados e um circuito é proposto (secção 4.1), que exemplifica como o output da metodologia pode ser útil como base para estudos mais abrangentes. Na secção 4.2 uma situação particular do caso de estudo é debatida, que mostra como ajustar as metodologias para lidar com situações casuísticas, específicas da cidade em estudo. Finalmente, na secção 5 são apresentadas conclusões e linhas de futura pesquisa.

Esta pesquisa usou como ambiente SIG o ESRI ArcGIS Desktop e a sua extensão, o Network Analyst. Faz-se notar também que o caso de estudo 
lidou essencialmente com a parte central da cidade de Coimbra (designada como "área de estudo"), com particular ênfase na parte da cidade a leste do Rio Mondego, que é onde a maior parte da população vive.

\section{TRATAMENTO EM AMBIENTE SIG DA REDE VIÁRIA}

Como argumentado acima, o relevo é um fator importante para quem viaja de bicicleta, que condiciona o uso massivo desta como modo de transporte. Declives maiores do que 5\% são indesejáveis não só porque as subidas são difíceis de transpor para muitos ciclistas, mas também porque as descidas requerem um controle apertado da velocidade por parte destes. Como exemplo da dificuldade associada às subidas, considere-se um caminho com 7\% de inclinação. De acordo com a análise de regressão de Parkin \& Rotherham (2010), tal subida é feita, em média, à velocidade de $3,2 \mathrm{~m} / \mathrm{s}$ e transmitindo o ciclista ao pedal uma potência mecânica de 261 W. Se olharmos à parte desta potência dedicada associada à energia potencial gravítica,

$$
P_{g}=M g v s
$$

vemos que para subir uma inclinação de 7\% à velocidade de 3,2 m/s, e assumindo uma massa total ciclista-bicicleta de $95 \mathrm{~kg}$, são necessários 209 W para vencer a gravidade o que, para uma eficiência mecânica da bicicleta de 95\%, representa nada menos do que $84 \%$ da potência mecânica desenvolvida pelo ciclista no pedal.

Independentemente dos números acima, na prática a perceção humana do esforço despendido é também importante. Por essa razão, tanto a Austroroads como a American Association of State Highway and Transportation Officials (AASHTO) propuseram valores desejáveis para declives, que salvaguardem o conforto do ciclista (AASHTO, 1999; AUSTROROADS, 2009). Na tabela abaixo temos as diretrizes AASHTO para declives e respetivos comprimentos máximos aceitáveis. 
Tabela 1

\begin{tabular}{|c|c|}
\hline Declive & Comprimento aceitável \\
\hline $5-6 \%$ & $240 \mathrm{~m}$ \\
\hline $7 \%$ & $120 \mathrm{~m}$ \\
\hline $8 \%$ & $90 \mathrm{~m}$ \\
\hline $9 \%$ & $60 \mathrm{~m}$ \\
\hline $10 \%$ & $30 \mathrm{~m}$ \\
\hline $11+\%$ & $15 \mathrm{~m}$ \\
\hline
\end{tabular}

\subsection{Análise de ciclabilidade}

A primeira tarefa é pois classificar os arcos da rede viária, no que respeita ao seu declive e comprimento. Antes disso, porém, apresentam-se abaixo alguns detalhes técnicos importantes para o efeito.

Em primeiro lugar é conveniente colocar a rede em formato $3 \mathrm{D}$ com precisão suficiente. No caso de estudo foi usado um modelo digital de superfície (MDS) com 1 metro de resolução, usando a ferramenta GIS interpolate shape (e também a package ETGeoWizards).

No ambiente ArcGIS uma rede viária é modelada da seguinte forma: o arco entre dois nós, A e B, é modelado como uma linha poligonal, definida pelas coordenadas de uma sequência de vértices (neste caso 3D) cujo primeiro e último se designam respetivamente por "From" e "To". Ao nível do Network Analyst existem dois sentidos ("From-To" e “To- From"), o que faz da rede uma rede orientada. Apesar da maioria das ruas terem declives aproximadamente constantes na sua extensão, há casos em que não é assim. Como tal, e na metodologia seguida, cada arco é decomposto, em relação ao seu sentido de referência, em três tipos de segmentos: subida, descida e plano. Como em cada segmento o declive é constante (a representação em SIG do arco é uma linha poligonal), atendendo a (1) vemos que segmentos de cada tipo podem ser agregados numa só parte, com comprimento igual à soma dos comprimentos das componentes e declive igual à média dos declives, ponderada pelos comprimentos das respetivas projeções horizontais. 
Podemos pois caraterizar cada arco por $\left(l_{u p}, s_{u p}\right),\left(l_{\text {down }}, s_{\text {down }}\right)$ e $l_{o}$, onde $l_{\text {up }}\left(l_{\text {down }}\right)$ se referem aos comprimentos das partes ascendentes (descendentes), $s_{u p}\left(s_{\text {down }}\right)$ aos declives médios ascendentes (descendentes) e $l_{O}$, o comprimento plano. Claramente, o que é ascendente no sentido"From-To" torna-se descendente no sentido "To-From" e vice-versa. Todas estas grandezas foram calculadas usando um script auxiliar (em linguagem Python) desenvolvido especificamente para o efeito. $\mathrm{Na}$ realidade, e para o cálculo do declive de cada arco, o script considera uma sequência de pontos que pode ser fundamentalmente de 3 tipos:

1. Vértices do próprio arco.

2. Pontos virtuais à distância equivalente do tamanho da célula do raster, 1 metro para o DSM.

3. Pontos distanciados entre si a um valor a fornecer pelo utilizador.

As cotas dos pontos considerados nos três métodos foram calculadas por interpolação bilinear tendo em consideração a sua posição relativamente aos centros de células adjacentes, e as respetivas cotas, dadas pelo raster (MDS). O processo de determinação de declives foi relativamente complexo. Basicamente resultou da comparação dos resultados obtidos pelos três métodos, acoplado a um processo de triagem, chegando ao reajuste dos próprios vértices dos arcos, em particular para arcos pequenos. Pode dizer- se, porém que, para arcos grandes, o $3^{\circ}$ método, com os pontos virtuais distanciados entre si de $45 \mathrm{~m}$ ou, o que é o mesmo, considerando os arcos modelados por linhas poligonais com segmentos de $45 \mathrm{~m}$ foi, em geral, o método usado. Tal significa, grosso modo, que a $1 \%$ de declive, num segmento $(45 \mathrm{~m})$, corresponde uma diferença de cotas entre os seus extremos de $45 \mathrm{~cm}$. Ora, para o tipo de MDS utilizado, o indicador de qualidade desvio padrão é inferior a $15 \mathrm{~cm}$ (HUISING \& PEREIRA, 1998), mas o estudo apresentado em GONÇALVES et al. (2011), refere tanto para o desvio padrão como para a raíz do erro quadrático médio, $10 \mathrm{~cm}$, do que resulta $16,4 \mathrm{~cm}$ para um nível de confiança de $90 \%$. Portanto, podemos falar de 
precisão nos declives da ordem de 0,3\%. Porém, em virtude de provável cancelamento de erros, para arcos de grande comprimento, é de admitir erros inferiores. Outro aspecto considerado consistiu no facto de a esmagadora maioria dos arcos declivosos serem de tipo rampa linear, ou seja com decive aproximadamente constante ao logo do seu percurso, facto aliás vulgar, em particular nas zonas não muito antigas.

À partida poderia pensar-se que a energia potencial gravítica do conjunto homem- bicicleta associada a (1) poderia ser usada regenerativamente nas descidas. No entanto, na prática tal reaproveitamento nem sempre é possível. De facto, se a descida for muito longa ou muito abrupta, o ciclista terá de travar para evitar velocidades excessivas, e assim dissipar parte da energia potencial. A questão poderá ainda assim ser relevante em caminhos de perfil ondulado, com subidas e descidas curtas, principalmente se os declives andarem por volta de $6 \%$ (ou seja um pouco acima do que aqui se considera como fronteira para a ciclabilidade; ver mais adiante). Todavia, dado que a esmagadora maioria dos caminhos não tem tais perfis, a caraterização simples proposta no parágrafo acima parece ser uma aproximação razoável.

Na Figura 1 é apresentada uma análise de ciclabilidade dos arcos da rede correspondente à zona da cidade de Coimbra em estudo, tendo-se atribuído, no que toca aos declives, uma cor correspondente ao grau de ciclabilidade (o verde designa arcos cicláveis). Todos os declives de 0-5\% foram considerados cicláveis em ambos os sentidos. O pavimento das ruas, apesar de importante, especialmente para declives 3-5\%, é geralmente bom; como tal não foi considerado neste caso de estudo. $O$ resultado é ilustrado na Figura 1 abaixo (à direita), juntamente com uma representação da topografia (à esquerda).

Da Figura podem identificar-se desde logo algumas zona cicláveis, que correspondem à predominância de arcos verdes sobre áreas grandes. Outras partes da cidade têm igualmente arcos cicláveis, mas não é fácil de ver se esses arcos ligam zonas importantes. Assumindo, como mencionado acima, todos os declives 0-5\% como estritamente cicláveis 
(cicláveis em ambos os sentidos, independentemente do comprimento), uma inspeção revela que aproximadamente 53\% (\% de comprimento) da rede viária é ciclável. Isto inclui arcos isolados, mas exclui arcos com declive maior do que 5\% mas que ainda assim são cicláveis de acordo com a Tabela 1 (arcos aqui designados por fracamente cicláveis; 12\% do total). Os restantes arcos perfazem 35\% da rede viária e são não-cicláveis, no sentido em que não podem ser totalmente percorridos em pelo menos um dos sentidos, de acordo com a Tabela 1.
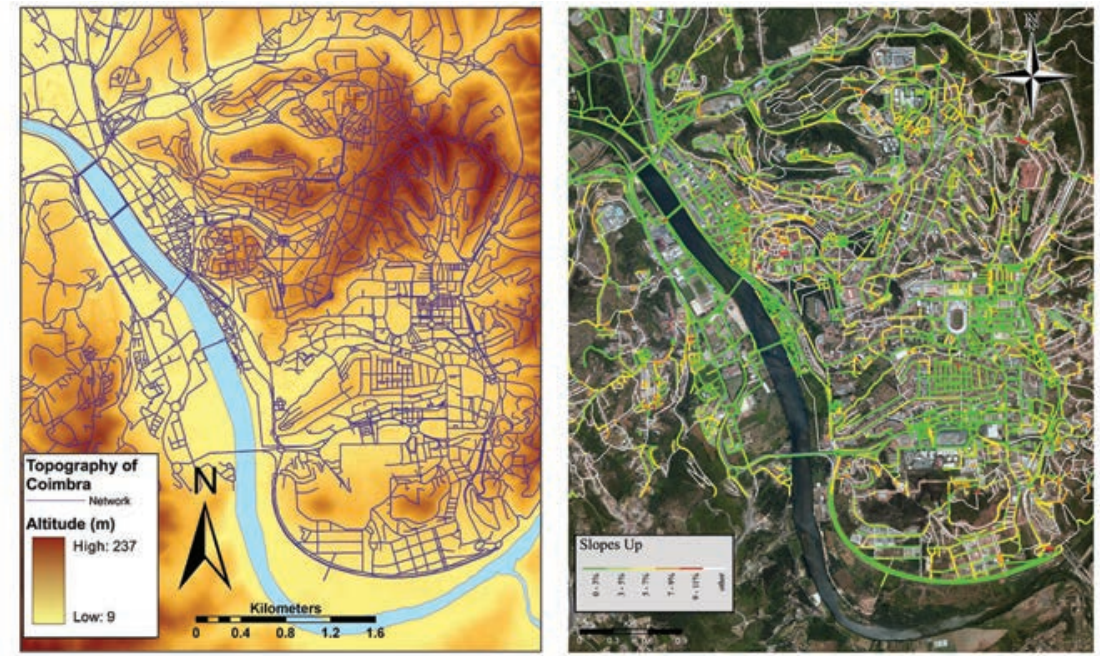

Figura 1 - Topografia de Coimbra e ciclabilidade da sua rede viária, de acordo com AASHTO (1999)

Chama-se aqui a atenção para um pormenor importante. Arcos adjacentes com declives de 5-11\% que sejam cicláveis podem originar um problema de cadeia. Ou seja, apesar de cada um destes arcos ser por si só ciclável, se vários deles formarem uma sequência, o comprimento $l_{u p}$ do caminho composto poderá exceder os limites de comprimento indicados na Tabela 1. Esta questão não foi considerada Figura 1, mas foi tomada em consideração nas análises abaixo, onde relevante.

\subsection{Permeabilidade}

Para analisar a permeabilidade, aqui definida como a extensão da cidade onde o transporte por bicicleta (no sentido do chamado "com- 
muting", movimentos pendulares) é possível, a ciclabilidade dos arcos não é suficiente. Um arco, ou pequeno grupo destes, pode estar rodeado de arcos não-cicláveis, o que torna a vizinhança não-contribuinte para a análise de permeabilidade. Isto é equivalente a dizer que definir buffers à volta de arcos cicláveis é insuficiente para a dita análise. Assim, enquanto o conceito de ciclabilidade é tomado aqui como referente a cada dos arcos, o conceito de permeabilidade diz mais respeito a zonas da rede. Por outro lado, uma zona permeável, para além de ter de permitir viagens longas sobre uma parte significativa da área sob estudo, deverá também permitir viagens em ambos os sentidos entre pares de pontos. No caso de uma cidade declivosa, em geral isso só será possível recorrendo a dispositivos de auxílio (p.ex. elevadores, funiculares, escadas/rampas rolantes). Tal leva naturalmente à questão de onde localizar tais dispositivos, por forma a minimizar o seu número, bem como a sua extensão. No entanto, a permeabilidade tem subjacente aquilo que se entende por arco ciclável. Por exemplo, se para a ciclabilidade se considerarem somente Os arcos completamente cicláveis, teremos um certo conjunto de zonas de permeabilidade; se se considerar por ciclável um arco em que $\left(l_{u p}, s_{u p}\right)$ respeita a Tabela 1 , e $s_{\text {down }} \leq 11 \%$, poderemos ter outras zonas de permeabilidade.

\section{LOCALIZAÇÃo DE Dispositivos de AUXílio}

A metodologia consiste em vários passos.

No primeiro, é feita uma análise ao relevo, com vista a identificar um conjunto de pontos representativos da zona em estudo. Estes pontos estão normalmente localizados em zonas de planalto ou crista, mas também nas zonas baixas (designamos este pontos por 'pontos de planalto'). O conjunto escolhido para o caso de estudo está representado na Figura 2 abaixo. O local mais alto da cidade, bem com o mais baixo, devem estar neste conjunto, ou perto de um ponto do mesmo.

No segundo passo, a rede é configurada (no contexto do Network Analyst) tal que todos os arcos não estritamente cicláveis fiquem, pelo uso de uma "restriction", "Prohibited" em ambos os sentidos (recorde-se que arcos estritamente cicláveis têm $\left.\left(l_{u p}, s_{u p}\right) \leq 5 \%\right)$. Vedaram-se também alguns arcos impróprios para a bicicleta (algumas pontes e vias rápidas). 


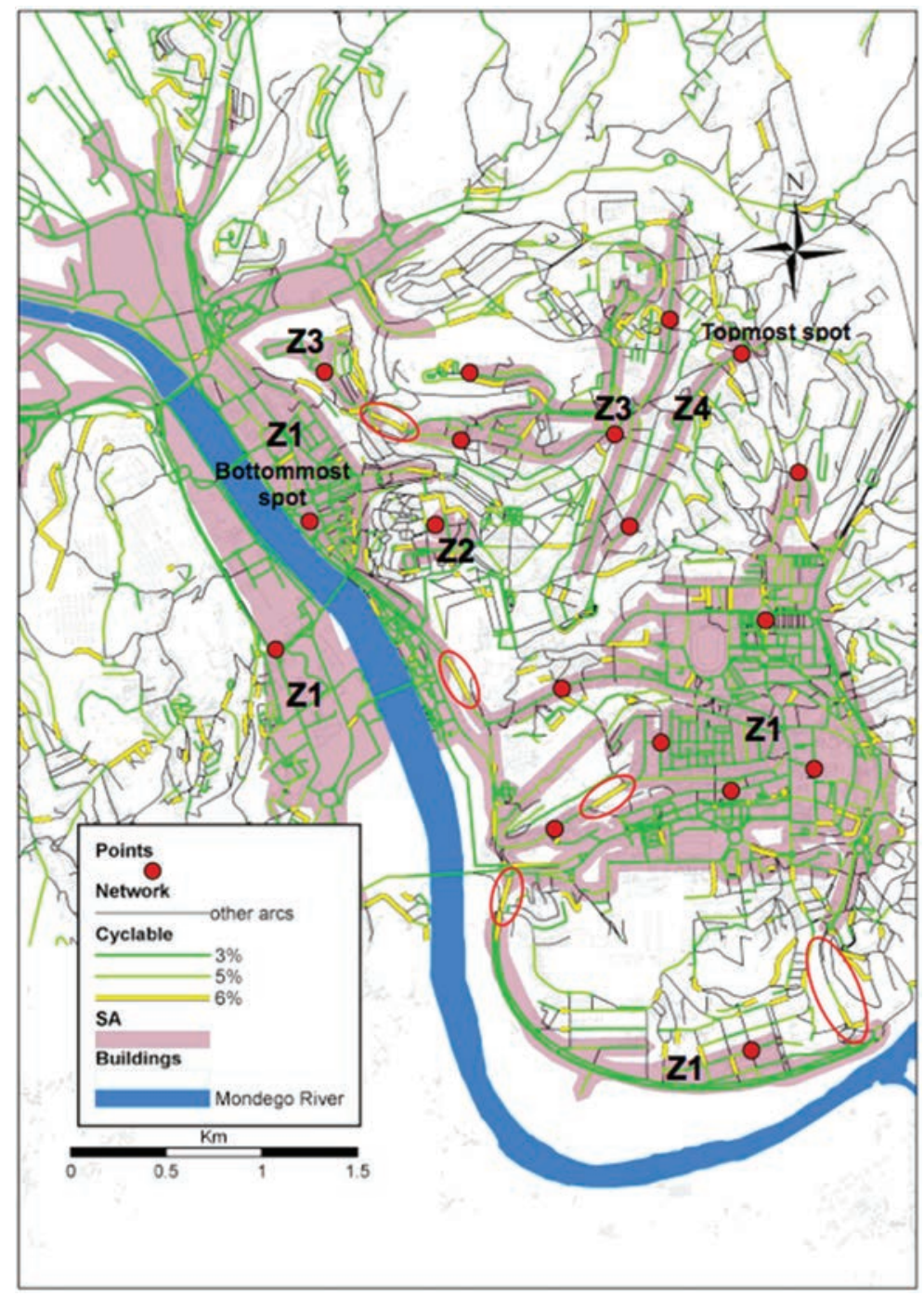

Figura 2 - Área de serviço aos pontos de planalto - desconexões

No terceiro passo a área de serviço (AS) relativa ao conjunto de pontos de planalto é obtida. Considerou-se para o efeito um "Default Break" grande (infinito em teoria, na prática quilómetros q.b.), o que leva a AS a estender-se até nodos da rede onde encontre arcos restritos. Para efeitos de visualização, usou-se um buffer ("Trim Polygons") de 50 $\mathrm{m}$, valor de compromisso entre a visibilidade gráfica e a visualização de desconexões entre sub-AS geradas (sombreados na Figura 2), e 
que permite também uma determinação aproximada das populações envolvidas. A AS está representada na Figura 2.

Juntamente com as áreas de serviço, a Figura 2 indica também, a amarelo, arcos com $5 \% \leq\left(s_{u p}, s_{\text {down }}\right) \leq 6 \%$ e $\left(l_{u p}, l_{\text {down }}\right) \leq 240 \mathrm{~m}$. A relevância destes arcos para a conexão entre zonas torna alguns deles potenciais candidatos para melhorias infraestruturais, com especial destaque para os quatro caminhos indicados por círculos a vermelho na Figura 2. Estes caminhos, que respeitam a Tabela 1, são essenciais para ligar algumas zonas e não formam cadeias.

Dado que os locais mais alto e mais baixo da cidade estão na AS obtida, é claro que, a menos de situações de sela/declive excessivo, se fosse possível preencher as desconexões, toda a área de estudo (ou pelo menos grande parte dela) tornar-se-ia permeável. O último passo consiste então numa análise detalhada dos arcos situados nas desconexões.

Inspecionando as AS e caminhos a amarelo, verifica-se que uma parte substancial do Sul da cidade e margem ribeirinha pode ser feita permeável (zona Z1, "Baixa”). Note-se que é irrelevante se a AS é obtida "towards" ou "away" dos pontos, uma vez que os arcos não proibidos são cicláveis em ambos os sentidos.

Encontrar caminhos orientados ligando duas sub-AS pode ser feito da seguinte forma sistemática. Dado que dentro de cada sub-AS todos os arcos são estritamente cicláveis, use-se a opção de routing do Network Analyst e considere-se dois pontos, $\mathrm{X}$ e Y, em sub-SA diferentes. Proíbase $\operatorname{arcos} \operatorname{com}\left(s_{u p}, l_{u p}\right)$ fora da Tabela 1 e arcos com $s_{\text {down }} 11 \%$. Defina-se classes de declives, p.ex. 0-5\%, 5-6\%, 6-8\%, 8-11\% (ascendente) e $\leq 11 \%$ (descendente) e $0 \%$, e encontre-se os trajetos mais curtos entre $\mathrm{X}$ e $\mathrm{Y}$, e entre $\mathrm{Y}$ e $\mathrm{X}$, usando a função de desutilidade

$$
w_{1} \Sigma_{j} l_{j}^{(1)}+w_{2} \Sigma_{j} l_{j}^{(2)}+\cdots+w_{N} \Sigma_{j} l_{j}^{(N)}
$$

onde $\mathrm{N}$ é o numero de classes, $w_{i}$ o peso para componentes da classe $i$ e $\sum_{j} l_{j}(i)$ o comprimento dos componentes da classe $i$. Os pesos $w_{i}$ funcionam aqui como um filtro, dado que valores mais baixos aumentam 
a preferência pelas componentes da classe respetiva. Esta propriedade pode ser usada para encontrar conexões com declives 0-6\% ou para tentar evitar o problema da cadeia (atribuindo pesos mais altos às componentes de maior declive). Se este procedimento conseguir encontrar dois caminhos, um em cada sentido, entre as sub-AS, então uma conexão entre estas é estabelecida. Eventualmente toda a zona entre as sub-AS fica conectada, a menos de situações de sela/declives excessivos (o que em geral não é o caso). O procedimento pode ser repetido para outros pontos $\mathrm{X}$ e $\mathrm{Y}$ das sub-AS em locais onde estas se aproximem uma da outra. Os trajetos assim obtidos podem subsequentemente ser estudados do ponto de vista de engenharia, caso seja necessário intervir, p.ex. para melhorar o pavimento ou colocar dispositivos de auxílio com vista a selecionar uma solução para implementação.

Usando o procedimento acima foi possível encontrar uma conexão para a sub-AS mais a sul (ver Figura 3). Esta sub-AS pode ser acedida por uma conexão ciclável nos dois sentidos a oeste, perto do rio, e por um caminho no lado nascente, este apenas no sentido norte-sul (círculo vermelho mais à direita na Figura 2). Esta última permitiu incluir na zona permeável uma urbanização perto dela.

Não foi, no entanto, possível encontrar quaisquer conexões entre três zonas, Z2, Z3 e Z4 ("Alta"), que se destacam claramente como regiões desconexas. Claramente, não há forma de ligar as quatro zonas cicláveis, dado que não é possível circular em ambos os sentidos sem a ajuda de dispositivos de auxílio.

A área de serviço serve uma população de cerca de 37000 habitantes, para um total de 60000 residentes na área de estudo. A maioria destes, 26 000, vivem em Z1, desligados dos 11000 de Z3 e Z4.

No caso de estudo, a conectividade entre zonas pode ser restabelecida através de três dispositivos (Figura 3, da esquerda para a direita): dispositivo 1: Z1-Z3, dispositivo 2: Z3- Z4, dispositivo 3: Z1-Z4. A zona Z2 tem detalhes próprios e será analisada numa secção dedicada. Dos três dispositivos, apenas o dispositivo 3 é indispensável, visto que leva até ao local mais alto da cidade. 
$\mathrm{Na}$ escolha das localizações deve ser dada prioridade à transposição de declives tão elevados quanto possível, dado que isso conduz normalmente a dispositivos de menor extensão. Uma forma de o conseguir consiste em alinhar os dispositivos ao longo de arcos existentes, usando a opção routing do Network Analyst e uma técnica semelhante à da secção 2.2 para encontrar caminhos de conexão, desta feita com função desutilidade dada por uma combinação convexa de $1 / s_{u p}$ $\mathrm{e} \mathrm{L}=l_{u p}+l_{\text {down }}+l_{O}$ (se $s_{u p} \approx 0$ atribui-se-lhe um valor artificial). Outra possibilidade é usar (eventualmente construir) um novo caminho, não existente na rede. No caso de estudo usou-se a primeira forma para obter a localização dos dispositivos 2 e 3, e a segunda para o dispositivo 1. Mais dispositivos poderiam ser considerados, se se desejasse encurtar mais as distâncias.

Resolvidas as conexões entre sub-SA, os arcos orientados pertencentes a essas conexões devem ser marcados como cicláveis.

\section{RESUltados PARA COIMBRA}

As secções 2 e 3, ao terem já sido apresentadas no quadro do caso de estudo, contém já parte dos resultados para Coimbra. Nesta secção estendem-se esses resultados no sentido de um eventual circuito de bicicleta para a cidade e analisa-se em detalhe as particularidades da zona Z2 (Pólo I da Universidade).

\subsection{Um possível circuito de bicicleta}

De acordo com Pucher (1997), é por vezes útil considerar um circuito de bicicleta dentro da área de estudo, ciclável em ambos os sentidos. Desta forma, um ciclista que se queira deslocar entre dois quaisquer pontos da cidade poderia (1) dirigir-se ao circuito, (2) percorrer parte deste, (3) sair dele em direção ao seu destino. Tal circuito deve ser particularmente adequado ao trânsito velocipédico e prolífico em dispositivos de auxílio. Outro aspeto do circuito, aliás já referido, é que deverá dar acesso aos pontos mais alto e mais baixo da cidade. Isto porque, excluindo situações de sela e/ou declives excessivos, todos 
os pontos da área de estudo são acessíveis a partir do local mais alto. O local mais baixo é importante porque este é, novamente, a menos de situações de sela/declives excessivos, acessível a partir de todos os pontos e, dado que liga (via circuito) ao local mais alto, conecta assim uma parte significativa dos pontos da área de estudo. É o que acontece no caso de estudo, com pequenas exceções, a mais importante sendo a colina que alberga o Pólo I da Universidade, Património Mundial UNESCO (zona Z2, ver Figura 2).

Os três dispositivos localizados pelo método descrito na secção 3 sugerem um circuito em forma de anel, plenamente ciclável. Esse possível circuito está ilustrado na Figura 3.

A principal caraterística do circuito é ser ciclável em ambos os sentidos, pelo que a AS do circuito é também a AS de um qualquer dos seus pontos. Essa AS corresponde à zona permeável final da área sob estudo. Note-se no entanto que o problema das cadeias dificulta a obtenção exata dessa AS. Apesar disso, é possível obter uma "zona de permeabilidade mínima", abrindo arcos com $s_{u p} \leq 5 \%$ e $s_{\text {down }} \leq 11 \%$ (além dos do circuito e os conectando sub-SA). A AS resultante está na Figura 3 , tirada a partir do ponto a vermelho. Abrindo os arcos orientados com $\left(l_{u p}, s_{u p}\right)$ na Tabela 1 e $s_{\text {down }} \leq 11 \%$ leva a uma "zona de permeabilidade máxima", que curiosamente não difere muito da zona mínima (daí não se apresentar).

Não há muitas alternativas para o circuito nas suas partes norte, sul e este. Já a parte oeste (e noroeste), que cruza o centro da cidade, pode ser alvo de um estudo mais detalhado. Tal estudo cai no entanto fora do âmbito desta comunicação, cujo objetivo é apresentar a metodologia acima. Refira-se que é conveniente dotar o circuito de atributos amigos da bicicleta, tais como faixas adequadas, semaforização, regras de prioridade, boa pavimentação e baixa densidade de cruzamentos (c.f. AASHTO, 1999; CROW, 2007; WANG et al., 2008; AUSTROROADS, 2009; BROACH et al., 2012).

Um estudo populacional revela que cerca de 51000 habitantes beneficiariam do circuito. Além de conectar os 37000 habitantes de Z1, Z3 e Z4, o circuito alargaria a permeabilidade ciclável a mais 14000 
habitantes.

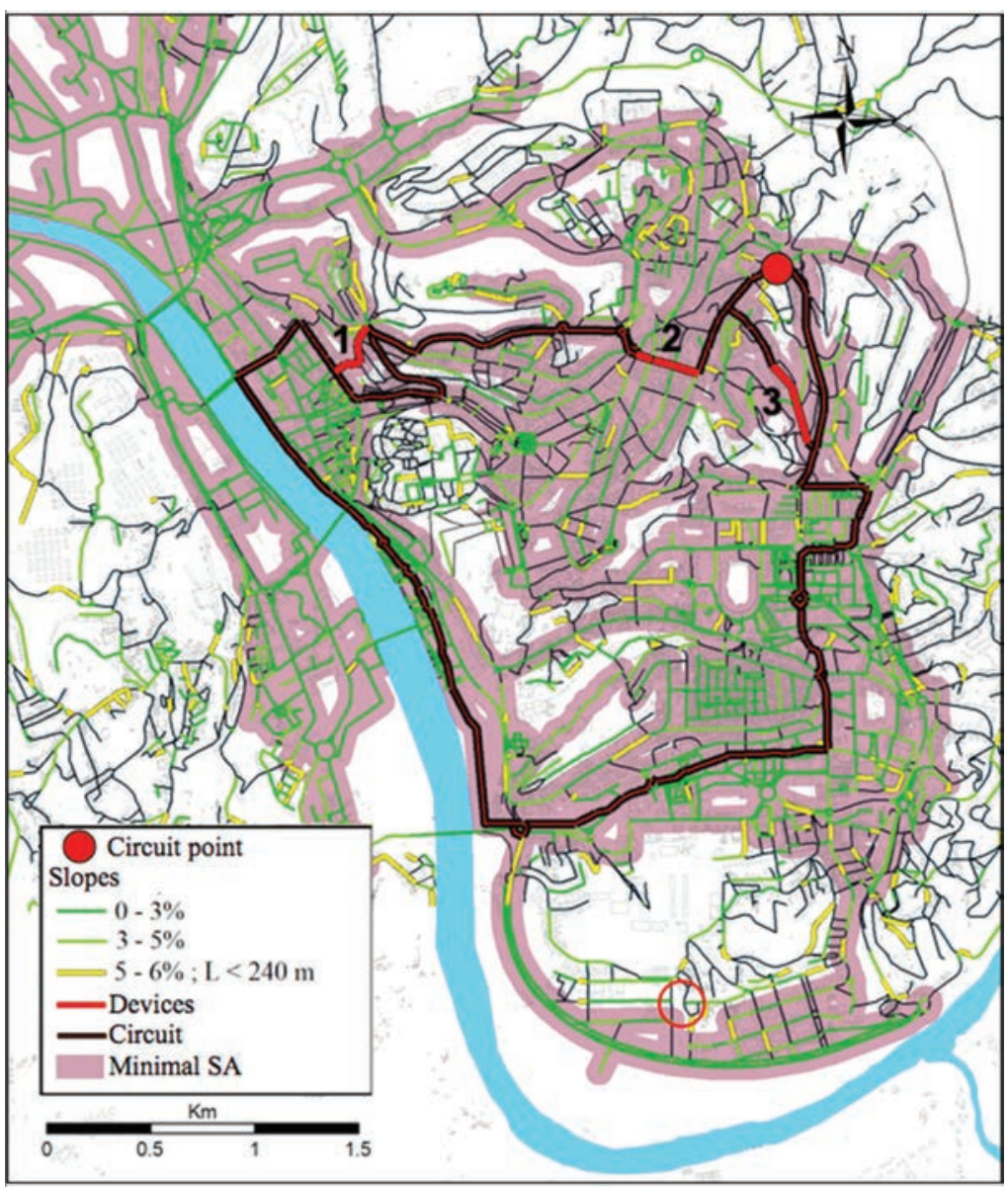

Figura 3 - Circuito ciclável e zona permeável final

\subsection{Ligação do Pólo I da Universidade}

Como se vê da Figura 2, um ponto de planalto localizado no Pólo I da Universidade gera uma pequena área de serviço, Z2, desconexa do resto da cidade, mesmo com os três dispositivos de auxílio considerados na secção 3 . Isto deve-se a uma situação de sela entre o local mais alto da cidade e Z2. Dado que esta zona está fora do circuito da secção 4.1, é interessante estudar a sua conexão sem considerar mais dispositivos.

Uma possibilidade de alcançar Z2 será usando um elevador já exis- 
tente, junto ao Mercado Municipal, elevador esse que está na zona de permeabilidade. O resto de $\mathrm{Z} 2$ tem caminhos descendentes de $s_{\text {down }} \leq$ 11\% e é plenamente ciclável nesse sentido. Ciclistas que se dirijam a Z2 vindos da "Alta" podem usar o elevador ou, em alternativa, um caminho de declive $7.5 \%, 220 \mathrm{~m}$ a sudeste de Z2 (este caminho situa-se no fundo da sela). Apesar deste caminho não obedecer à Tabela 1, pode eventualmente ser considerado como ciclável dado que o Pólo I é um destino procurado principalmente por ciclistas jovens. Ciclistas em trânsito de Z2 para a "Alta" têm de passar por dois arcos: 6\%/158 m e 6.8\%/116 m. Ambos obedecem à Tabela 1 e não formam uma cadeia, sendo por isso cicláveis em ambos os sentidos. Independentemente da forma de alcançar Z2, é de considerar colocar-se parques de estacionamento de bicicletas junto ao elevador do Mercado Municipal e do caminho de $7.5 \% / 220 \mathrm{~m}$, e assumir que ciclistas percorrem o resto a pé.

Como referido, Z2 é geralmente ciclável em sentido descendente, mas não em sentido ascendente. Elevadores, para além de fechar desconexões entre sub-AS mitigariam esta inconveniência. É de referir a conveniência em que pelo menos e principalmente o dispositivo 3 possa ser usado por peões, dado este ser um excelente atalho entre a parte sudeste da área de estudo e a parte norte, onde se situa o Hospital Universitário, juntamente com outros equipamentos de saúde.

\section{CONCLUSÕES}

Nesta pesquisa apresenta-se uma metodologia para, com recurso à tecnologia SIG, proceder não só ao estudo da ciclabilidade e da permeabilidade ciclável de uma cidade declivosa mas também, e principalmente, para a localização de dispositivos mecânicos de auxílio ao ciclista, com vista à melhoria da sua permeabilidade ciclável. Esta metodologia é genérica e pode ser aplicada a qualquer cidade, auxiliando assim os decisores a planear a sua cidade para a bicicleta. Por outro lado, permite e identificação de possíveis melhorias, elementos importantes em qualquer estudo a priori de permeabilidade ciclável. A metodologia de localização apresentada constitui, tanto quanto é do nosso conheci- 
mento, a primeira abordagem sistemática ao assunto.

Como pesquisa futura, seria interessante definir geradores e atratores de tráfego velocipédico e calcular tempos de viagem entre ambos, com e sem dispositivos de auxílio e/ou circuito. A metodologia pode também ser adaptada a um sem-número de outras situações, tais como velocípedes eletricamente assistidos. Possivelmente para estes velocípedes os dispositivos de auxílio seriam desnecessários, tornando-se interessante comparar ambos os cenários no que respeita a custos económicos e energéticos. Outra possibilidade seria estudar cidades cuja rede viária contenha muitos caminhos com pavimento degradado (independentemente do relevo). Para tal bastaria configurar o SIG de maneira a modelar os arcos correspondentes através de declives artificiais e subsequentemente gerar áreas de serviço. O resultado mostraria então quais os caminhos prioritários para uma intervenção que restabeleça a conectividade.

\section{AGRADECIMENTOS}

Este trabalho foi parcialmente financiado pela Fundação Portuguesa para a Ciência e Tecnologia (FCT) sob a Refa "PEst-OE/EEI/UI308/2014", pelo projeto Energy and Mobility for Sustainable Regions (EMSURE) - Ref ${ }^{a}$ CENTRO-07-0224-FEDER-002004, e enquadra-se na iniciativa Energy for Sustainability da Universidade de Coimbra.

\section{BIBLIOGRAFIA}

AASHTO (1999). AASHTO Guide for the Development of Bicycle Facilities. American Association of State Highway and Transportation Officials, EUA. ISBN 1560511028. Disponível online no endereço url: http://www. industrializedcyclist.com/aashto.pdf (acedido em 30 março, 2014).

AUSTROADS (2009) The Guide to Road Design - Part 6A: Pedestrian and Cyclist Paths. Austroads Publications, No AGRD06A-09, 115 p. ISBN 9781921551819.

BROACH, J et al. (2012) - "Where do cyclists ride? A route choice model developed with revealed preference GPS data." Transportation Research Part A, Vol. 46, 1730- 1740. http://dx.doi.org/10.1016/j.tra.2012.07.005 
BYPAD (2008) Bicycle Policy Audit. Projeto da Comissão Europeia. Disponível online no endereço url: http://www.bypad.org/cms_site.phtml?id= 552\&sprache $=$ en (acedido em 30 março, 2014).

CERVERO, R et al. (2012) - "Bike-and-Ride: Build It and They Will Come." Working paper. Institute of Transportation Studies, University of California (ed.), Berkley, USA. Disponível online no endereço url: http://its.berkeley.edu/ publications/UCB/2012/VWP/UCB-ITS-VWP-2012-5.pdf (acedido em 30 março, 2014).

COMISSÃO EUROPEIA (2000) Cidades para Bicicletas, Cidades de Futuro. DireçãoGeral do Ambiente da Comissão Europeia. Disponível online no endereço url: http://ec.europa.eu/environment/archives/cycling/cycling_pt.pdf (acedido em 30 março, 2014).

CROW (2007). Design manual for bicycle traffic. CROW, The Netherlands, 388 p. ISBN 9789066284944.

GONÇALVES, G et al. (2011) - "Avaliação da qualidade de modelos digitais de terreno obtidos por Lidar em ambiente urbano: um caso de estudo". VII Conferência Nacional de Cartografia e Geodesia, Lisboa, 5 e 6 de Maio.

HUISING, E \& PEREIRA, L (1998) - "Errors and accuracy estimates of laser data acquired by various laser scanning systems for topographic applications." ISPRS Journal of Photogrammetry and Remote Sensing, Vol. 53, No 5, 245-261.

PARKIN, J \& KOOREY, G (2012) - "Network planning and infrastructure design." Capítulo $6 \mathrm{em}$ Cycling and Sustainability I. Emerald Group Publishing, 131-160. ISBN 9781780522982.

PARKIN, J \& ROTHERHAM, J (2010) - "Design speeds and acceleration characteristics of bicycle traffic for use in planning, design and appraisal." Transport Policy, Vol. 17, 335-341. http://dx.doi.org/10.1016/j.tranpol.2010.03.001

PARKIN, J et al. (2008) - "Estimation of the Determinants of Bicycle Mode Share for the Journey to Work using Census Data." Transportation, Vol. 35, 93109. http://EconPapers.repec.org/RePEc:kap:transp:v:35:y:2008:i:1:p:93-109

PARKIN, J et al. (2007) - "Models of perceived cycling risk and route acceptability." Accident Analysis and Prevention, Vol. 39, 364-371. http://dx.doi. org/10.1016/j.aap.2006.08.007

PUCHER, J et al. (2011) - "Bicycling renaissance in North America? An update and re- appraisal of cycling trends and policies." Transportation Research Part A, Vol. 45, 451-475. http://dx.doi.org/10.1016/j.tra.2011.03.001

PUCHER, J \& BUEHLER, R (2008) - "Making Cycling Irresistible: Lessons from The Netherlands, Denmark and Germany." Transport Reviews, Vol. 28, N 4, 495-528. http://dx.doi.org/10.1080/01441640701806612

PUCHER, J et al. (1999) - "Bicycling renaissance in North America?: Recent trends and alternative policies to promote bicycling." Transportation 
Research Part A, Vol. 33, No 7-8, 625-654. http://dx.doi.org/10.1016/S09658564(99)00010-5

PUCHER, J (1997) - "Bicycling Boom in Germany: A Revival Engineered by Public Policy." Transportation Quartely, Vol. 51, No 4, 31-46.

RIETVELD, P \& DANIEL, V (2004) - "Determinants of bicycle use: do municipal policies matter?" Transportation Research Part A, Vol. 38, 531-550. http:// dx.doi.org/10.1016/j.tra.2004.05.003

SUZUKI, K et al. (2012) - "Proposal and Application of a New Method for Bicycle Network Planning." Procedia - Social and Behavioral Sciences, Vol. 43, 558570. http://dx.doi.org/10.1016/j.sbspro.2012.04.129

WARDMAN, M et al. (2007) - "Factors influencing the propensity to cycle to work." Transportation Research Part A, Vol. 41, No 4, 339-350. http://dx.doi. org/10.1016/j.tra.2006.09.011

WANG et al. (2008) - "Research on bicycle conversion factors." Transportation Research Part A, Vol. 42, 1129-1139. http://dx.doi.org/10.1016/j.tra. 2008.03.016

WOODCOCK et al. (2007) - "Energy and transport." The Lancet, Vol. 370, N 9592, 1078- 1088. http://dx.doi.org/10.1016/S0140-6736(07)61254-9 
Série Documentos

Imprensa da Universidade de Coimbra

Coimbra University Press

2015

- U M

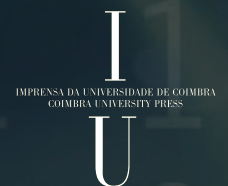

